\title{
Homotopy Analysis Solution for Magnetohydrodynamic Squeezing Flow in Porous Medium
}

\author{
Inayat Ullah, ${ }^{1,2}$ M. T. Rahim, ${ }^{2}$ Hamid Khan, ${ }^{2}$ and Mubashir Qayyum ${ }^{2}$ \\ ${ }^{1}$ Department of Mathematics, Edwardes College, Peshawar 25000, Pakistan \\ ${ }^{2}$ Department of Mathematics, National University of Computer and Emerging Sciences, FAST, Peshawar 25000, Pakistan \\ Correspondence should be addressed to Inayat Ullah; p119952@nu.edu.pk
}

Received 31 March 2016; Revised 7 May 2016; Accepted 10 May 2016

Academic Editor: Ricardo Weder

Copyright (c) 2016 Inayat Ullah et al. This is an open access article distributed under the Creative Commons Attribution License, which permits unrestricted use, distribution, and reproduction in any medium, provided the original work is properly cited.

\begin{abstract}
The aim of the present work is to analyze the magnetohydrodynamic (MHD) squeezing flow through porous medium using homotopy analysis method (HAM). Fourth-order boundary value problem is modeled through stream function $\psi(r, z)$ and transformation $\psi(r, z)=r^{2} f(z)$. Absolute residuals are used to check the efficiency and consistency of HAM. Other analytical techniques are compared with the present work. It is shown that results of good agreement can be obtained by choosing a suitable value of convergence control parameter $h$ in the valid region $R_{h}$. The influence of different parameters on the flow is argued theoretically as well as graphically.
\end{abstract}

\section{Introduction}

The squeezing movement normal to two plates is observed in many hydromechanical devices such as motors, engines, and hydraulic lifters, where compression/injection processes using pistons and clutches are found. Due to the utility of these devices, significant research effort is being made for their improvement. Other industrial applications include polymer processing, while medical applications include the modeling of synthetics transportation inside living bodies. As such, the study of squeezing effect, in addition to other properties such as magnetohydrodynamics (MHD) and porosity, has become one of the most active topics in fluid mechanics. The study of porosity effects after introduction of the modified Darcy Law [1] specifically contributed to oil and gas production industry, detection of ground water pollution due to leakage of chemicals from tanks and oil pipelines, ground water hydrology, and recovery of crude oil from pores of reservoir rocks [2-5]. These contributions and others $[6,7]$ are generally found in reservoir, chemical, civil, environmental, agricultural, and biomedical engineering.

These studies are typically modeled using small parameters in nonlinear differential equations which are expressed as series expansions. The exact solution using perturbation methods is therefore not always possible and this poses a considerable challenge to researchers. A recently developed analytic method known as homotopy analysis method (HAM) by Liao in 1992, however, has given promising results as it does not require modeling of the small parameter [8]. In fact, in quite contrast, HAM provides a way to accelerate the series solution conversion in the form of auxiliary parameter. The equations are reduced by HAM to a set of linear ordinary differential equations based on the homotopy of topology. These sets of equations can then be computed by mathematical software like Mathematica, Maple, MATLAB, Octave, SageMath, or Maxima. Applications are found in various problems of science and engineering such as expression of skin friction coefficient and reduced Nusselt and Sherwood numbers [9-12]. Analytical tools like homotopy perturbation method (HPM), $\delta$-expansion method, and Adomian decomposition method (ADM) are special cases of HAM [13].

Mabood and Khan [14,15] successfully applied homotopy analysis method for the study of heat transfer on MHD stagnation point flow in porous medium and boundary layer flow and heat transfer over a permeable flat plate in a Darcian porous medium. Analytic solutions for unsteady twodimensional and axisymmetric flows were presented by Rashidi et al. [16]. The study of heat and mass transfer in the 
context of squeezing flow was performed by Mustafa et al. [17]. Kirubhashankar and Ganesh [18] analyzed electrically conducting MHD viscous flows. A two-dimensional MHD problem was approximated using homotopy perturbation method (HPM) by Siddiqui et al. [19, 20]. Unsteady flow of viscous fluid between porous plates is studied by Ganesh and Krishnambal [21] and Mohamed Ismail et al. [22]. Shevanian et al. $[23,24]$ successfully used HAM to study singular linear vibrational BVPs and MHD squeezing flow between two parallel disks. The same authors $[25,26]$ used predictor homotopy analysis method (PHAM) to investigate nonlinear reactive transport model and nanoboundary layer flows with nonlinear Navier boundary condition.

This work is an effort to investigate MHD squeezing flow of Newtonian fluid between two parallel plates passing through porous medium by homotopy analysis method. Using similarity transforms, the governing partial differential equations are converted to equivalent nonlinear ordinary differential equation and then solved using the mentioned scheme. Velocity profile of fluid is argued by varying various parameters involved.

\section{Mathematical Model}

In two dimensions, if cylindrical coordinates of the velocity $\mathbf{u}$ of moving plates are $\left[u_{r}, 0, u_{z}\right], \nabla$ is defined to be $(\partial / \partial r,(1 / r)(\partial / \partial \theta), \partial / \partial z), \omega=\nabla \times \mathbf{u}, \Omega(r, z)=\partial u_{z} / \partial r-$ $\partial u_{r} / \partial z, v=u_{r}^{2}+u_{z}^{2}$, and $\rho$, $p$, and $\kappa$ denote the density, pressure, and permeability, respectively, then, from momentum equation of steady squeezing flow in porous medium with MHD effect, $r, \theta$, and $z$ components are as follows.

$r$ component is as follows:

$$
\frac{\partial}{\partial r}(\rho v+p)-\rho \Omega u_{z}=-\left(\mu \frac{\partial \Omega}{\partial z}+A u_{r}\right)
$$

$\theta$ component is as follows:

$$
\begin{aligned}
\frac{1}{r} \frac{\partial p}{\partial \theta} & =0 \Longrightarrow \\
\frac{\partial p}{\partial \theta} & =0 \Longrightarrow \\
p & =f(r, z) .
\end{aligned}
$$

$z$ component is as follows:

$$
\frac{\partial}{\partial r}(\rho v+p)+\rho \Omega u_{r}=\frac{\mu}{r} \frac{\partial}{\partial r}(r \Omega)-\frac{\mu}{\kappa} u_{z}
$$

Here, $A=\mu / \kappa+\sigma B_{0}^{2}$ with $B_{0}$ as imposed magnetic field. Introducing stream function [27] $\psi(r, z)$ defined by

$$
\begin{aligned}
& u_{r}(r, z)=\frac{1}{r} \frac{\partial \psi}{\partial z} \\
& u_{z}(r, z)=-\frac{1}{r} \frac{\partial \psi}{\partial r}
\end{aligned}
$$

it can be easily proved that the continuity equation $\nabla \cdot \mathbf{u}=0$ is satisfied. With the help of $(4)$ and eliminating $(\partial / \partial r)(\rho v+p)$, (1) and (3) reduce to single PDE as follows:

$$
\begin{gathered}
\rho\left[\frac{\partial \psi}{\partial r} \frac{\partial}{\partial z}\left(\frac{\eta^{2} \psi}{r^{2}}\right)-\frac{\partial \psi}{\partial z} \frac{\partial}{\partial r}\left(\frac{\eta^{2} \psi}{r^{2}}\right)\right] \\
=-\frac{\mu}{r} \eta^{4} \psi+\frac{\mu}{\kappa} \eta^{2} \psi+\frac{\eta B_{0}^{2}}{r} \frac{\partial^{2} \psi}{\partial z^{2}} .
\end{gathered}
$$

Here, $\eta=\partial^{2} / \partial r^{2}-(1 / r)(\partial / \partial r)+\partial^{2} / \partial z^{2}$. If the moving plates are separated by distance $2 d$, then

$$
\begin{aligned}
u_{r} & =0, \\
u_{z} & =-v, \\
& \text { at } z=d, \\
u_{z} & =0, \\
\frac{\partial u_{r}}{\partial z} & =0
\end{aligned}
$$

$$
\text { at } z=0 \text {. }
$$

Using transformation $\psi(r, z)=r^{2} f(z)$, (5) reduces to single ODE as follows:

$$
\begin{gathered}
\frac{d^{4}}{d z^{4}} f(z)+\frac{2 \rho}{\mu} f(z) \frac{d^{3}}{d z^{3}} f(z)-\frac{1}{\kappa} \frac{d^{2}}{d z^{2}} f(z) \\
-\frac{\sigma B_{0}^{2}}{\mu} \frac{d^{2}}{d z^{2}} f(z)=0 .
\end{gathered}
$$

The boundary conditions in (6) are transformed to

$$
\begin{aligned}
& \frac{d^{2}}{d z^{2}} f(0)=0, \quad f(0)=0, \\
& \frac{d}{d z} f(d)=0, \quad f(d)=-\frac{u}{2} .
\end{aligned}
$$

Using nondimensional parameters $(2 / u) f^{*}=f, d z^{*}=z$, $(u / \mu) R_{m p}=\rho d, m_{h}=\sigma d B_{0} / \mu$, and $m_{p}=h / \kappa$ and omitting $*$ sign, (7) and (8) become

$$
\begin{aligned}
& \frac{d^{4}}{d z^{4}} f(z)+R_{m p} f(z) \frac{d^{3}}{d z^{3}} f(z)-m_{p} \frac{d^{2}}{d z^{2}} f(z) \\
& -m_{h} \frac{d^{2}}{d z^{2}} f(z)=0, \\
& \frac{d^{2}}{d z^{2}} f(0)=0, \quad f(0)=0, \\
& \frac{d}{d z} f(1)=0, \quad f(1)=1,
\end{aligned}
$$

where $R_{m p}$ is Reynold and $m_{h}, m_{p}$ are Hartmann numbers. 


\section{Application of HAM to Squeezing Flow}

HAM logically contains some analytic techniques such as Adomian's decomposition method, Lyapunov's artificial small parameter method, and $\delta$-expansion method. Thus, this technique can be regarded as a unified or generalized theory of these analytical techniques. Unlike other analytic techniques, the homotopy analysis method provides a simple way to control and adjust the convergence region and rate of solution series of nonlinear problems. Thus, this method is valid for nonlinear problems with strong nonlinearity. Homotopy analysis method provides great freedom to use base functions to express solutions of a nonlinear problem so that one can approximate a nonlinear problem more efficiently by means of better base functions [13].

In the present section, this technique is applied on (9) using boundary conditions (10). For solution expression polynomial base function $\left\{z^{2 n+1} \mid n=0,1,2,3, \ldots\right\}$ is used to determine $f(z)$ as follows:

$$
f(z)=\sum_{m=0}^{\infty} b_{n} z^{2 n+1},
$$

where $b_{n}$ are constants. By rule of solution expression and according to conditions in (10), the initial guess of the problem is

$$
f_{0}(z)=\frac{3 z-z^{3}}{2} .
$$

The auxiliary linear operator is chosen as

$$
L[f(z)]=\frac{d^{4}}{d z^{4}} f(z)
$$

with the property

$$
L\left(C_{1} \frac{z^{3}}{6}+C_{2} \frac{z^{2}}{2}+C_{3} z+C_{4}\right)=0 .
$$

Here, $C_{i}(i=1,2,3,4)$ are integration constants whose obtained values are

$$
\begin{aligned}
& C_{2}=C_{4}=0, \\
& C_{1}=-3, \\
& C_{3}=1.5 .
\end{aligned}
$$

By rule of solution expression in (2.51) and by (2.39) in [13], the auxiliary function $H(z)$ is chosen to be $H(z)=1$.

3.1. Zeroth-Order Deformation Equation. Using the homotopy introduced in [13], zeroth-order deformation equation is given by

$$
(1-q) L\left[\phi(z ; q)-f_{0}(z)\right]=q h H(z) N[\phi(z ; q)],
$$

where $N$ is a nonlinear operator defined by

$$
\begin{aligned}
N[\phi(z ; q)]= & \frac{d^{4} \phi(z ; q)}{d z^{4}}+R_{m p} \phi(z ; q) \frac{d^{3} \phi(z ; q)}{d z^{3}} \\
& -m_{p} \frac{d^{2} \phi(z ; q)}{d z^{2}}-m_{h} \frac{d^{2} \phi(z ; q)}{d z^{2}} .
\end{aligned}
$$

From here, the zeroth-order problem obtained is

$$
\begin{aligned}
(1-q) L\left[\phi(z ; q)-f_{0}(z)\right] & =q h H(z) N[\phi(z ; q)], \\
\frac{d^{2} \phi(0 ; q)}{d z^{2}} & =0, \quad \phi(0 ; q)=0, \\
\frac{d \phi(1 ; q)}{d z} & =0, \quad \phi(1 ; q)=1 .
\end{aligned}
$$

Here, $q$ is an embedding parameter and $h$ is a nonzero auxiliary parameter. It is observed that,

$$
\text { at } q=0 \text {, }
$$

$$
\phi(0 ; q)=f_{0}(z) ;
$$

at $q=1$,

$$
\phi(1 ; q)=f(z) .
$$

Hence, when $q$ varies from 0 to 1 , then $\phi(z ; q)$ varies from $f_{0}(z)$ to $f(z)$. By Maclaurin's expansion, $\phi(z ; q)$ can be expressed as

$$
\phi(z ; q)=f_{0}(z)+\sum_{n=1}^{\infty} f_{n}(z) q^{n} .
$$

The value of the auxiliary parameter $h$ is chosen in such a way that the series in (18) converges at $q=1$; that is,

$$
f(z)=f_{0}(z)+\sum_{n=1}^{\infty} f_{n}(z),
$$

where $f_{n}(z)=(1 / n !)\left(\partial^{n} f(z ; q) / \partial z^{n}\right) \|_{q=0}$.

3.2. nth-Order Deformation Equation. Differentiate (18) and (19) $n$-times with respect to $q$ and put $q=0$ to get $n$ th-order deformation equation as follows:

$$
\begin{aligned}
L\left[f_{n}(z)-\chi_{n} f_{n-1}(z)\right] & =h H(z) R_{n}\left(f_{n-1}\right), \\
\frac{d^{2} f_{n}(0)}{d z^{2}} & =0, \quad f_{n}(0)=0, \\
\frac{d f_{n}(1)}{d z} & =0, \quad f_{n}(1)=0 .
\end{aligned}
$$

Equation (24) reduces to

$$
f_{n}(z)=\chi_{n} f_{n-1}(z)+h L^{-1}\left[H(z) R_{n}\left(f_{n-1}\right)\right] \text {, }
$$


TABLE 1: Absolute residuals keeping $m_{h}=m_{p}=0.5$ and $R_{m p}=1$.

\begin{tabular}{|c|c|c|c|c|c|}
\hline \multicolumn{6}{|c|}{ Absolute residuals for different order HAM solutions } \\
\hline$z$ & 5 th order & 10th order & 15th order & 20th order & 25 th order \\
\hline 0.0 & 0 & 0 & 0 & 0 & 0 \\
\hline 0.1 & $9.5367 \times 10^{-9}$ & $7.7605 \times 10^{-14}$ & 0 & $5.5511 \times 10^{-17}$ & 0 \\
\hline 0.2 & $3.2067 \times 10^{-8}$ & $3.0953 \times 10^{-13}$ & $2.2210 \times 10^{-16}$ & $2.2210 \times 10^{-16}$ & $2.2210 \times 10^{-16}$ \\
\hline 0.3 & $1.6171 \times 10^{-7}$ & $9.2082 \times 10^{-13}$ & $4.4410 \times 10^{-16}$ & $2.2210 \times 10^{-16}$ & $2.2210 \times 10^{-16}$ \\
\hline 0.4 & $5.3675 \times 10^{-7}$ & $2.1336 \times 10^{-12}$ & 0 & 0 & 0 \\
\hline 0.5 & $1.3172 \times 10^{-6}$ & $3.5714 \times 10^{-12}$ & 0 & $4.4410 \times 10^{-16}$ & 0 \\
\hline 0.6 & $1.8889 \times 10^{-6}$ & $2.8302 \times 10^{-12}$ & $4.4410 \times 10^{-16}$ & $8.8820 \times 10^{-16}$ & $4.4410 \times 10^{-16}$ \\
\hline 0.7 & $2.0711 \times 10^{-7}$ & $5.1026 \times 10^{-12}$ & $4.4410 \times 10^{-16}$ & $4.4410 \times 10^{-16}$ & $4.4410 \times 10^{-16}$ \\
\hline 0.8 & $5.7686 \times 10^{-6}$ & $2.1931 \times 10^{-11}$ & 0 & $4.4410 \times 10^{-16}$ & $1.3323 \times 10^{-15}$ \\
\hline 0.9 & $1.3705 \times 10^{-5}$ & $3.2338 \times 10^{-11}$ & $4.4410 \times 10^{-16}$ & $8.8820 \times 10^{-16}$ & 0 \\
\hline 1.0 & $1.2902 \times 10^{-5}$ & $2.8715 \times 10^{-12}$ & $2.2205 \times 10^{-15}$ & $4.4410 \times 10^{-16}$ & $4.4410 \times 10^{-16}$ \\
\hline
\end{tabular}

where

$$
\begin{aligned}
L^{-1}= & C_{1} \frac{x^{3}}{6}+C_{2} \frac{x^{2}}{2}+C_{3} x+C_{4} \\
& +\iiint \int(\cdot) d z d z d z d z, \\
R_{n}\left(f_{n-1}\right)= & f_{n-1}^{i v}+R_{m p} \sum_{k=0}^{n-1} f_{k} f_{n-1-k}^{\prime \prime \prime} \\
& -\left(m_{h}+m_{p}\right) f_{n-1}^{\prime \prime},
\end{aligned}
$$

$$
f(z)=-\frac{e^{-\sqrt{M} z}\left(\sqrt{M} z\left(-e^{\sqrt{M} z}\right)-\sqrt{M} z e^{\sqrt{M} z+2 \sqrt{M}}+e^{2 \sqrt{M} z+\sqrt{M}}-e^{\sqrt{M}}\right)}{\sqrt{M} e^{2 \sqrt{M}}+\sqrt{M}-e^{2 \sqrt{M}}+1} .
$$

Here, $M=m_{h}+m_{p}$. Homotopy analysis solution is also derived in this case. The operator $N$ in (17) becomes

$$
\begin{aligned}
N[\phi(z ; q)]= & \frac{d^{4} \phi(z ; q)}{d z^{4}}-m_{p} \frac{d^{2} \phi(z ; q)}{d z^{2}} \\
& -m_{h} \frac{d^{2} \phi(z ; q)}{d z^{2}}
\end{aligned}
$$

and for $n$ th-order deformation equation $R_{n}$ in (28) becomes

$$
R_{n}\left(f_{n-1}\right)=f_{n-1}^{i v}-\left(m_{h}+m_{p}\right) f_{n-1}^{\prime \prime}
$$

In Table 4, comparison of exact solution with fifth- and tenthorder HAM solutions is made with the help of absolute error.

\section{Convergence of HAM Solution}

Solution obtained by homotopy analysis method in (23) contains auxiliary parameter $h$ which adjusts and controls the convergence. There is great freedom to choose the auxiliary parameter. For influence of $h$ on the solution, the convergence

$$
\chi_{n}= \begin{cases}0, & n \leq 0 \\ 1, & n>0\end{cases}
$$

\section{Exact Solution in Case of Zero Reynold Number}

In this section, a special case is studied when the Reynold number is zero and hence (9) becomes a linear differential equation. The exact solution obtained using boundary conditions in (10) is given by of $f^{(n)}(0)$, where $n$ is odd, is considered. The valid region $R_{h}$ of $h$ for which $f^{\prime}(0)$ converges is shown for different order solutions in Figure 1 . The curve $f^{\prime}(0)$ versus $h$ is said to be $h$-curve. From Figure 1 , it is observed that $R_{h}$ increases with the increase of approximation order. For fifth-order solution, the valid region for $h$ is $-1.6 \leq h \leq-0.3$. It is obvious from Figure 2 that when $m_{h}, m_{p}$ increase further, the valid region moves towards the right. Figures 3 and 4 are constructed to examine $R_{h}$ for increasing $m_{h}, m_{p}$, and $R_{m p}$.

\section{Results and Discussion}

Analytic solution, using homotopy analysis method, of magnetohydrodynamics squeezing flow through porous medium is studied. Four figures (Figures 1-4) are constructed, for various values of Reynold and Hartmann numbers, to examine the valid region $R_{h}$ which has a vital role in convergence of analytic solution. Table 1 shows different order absolute residuals for HAM solutions and it is clear to see that as the order of approximation increases further, the solution converges to exact solution. Fifth-order absolute residuals for various 


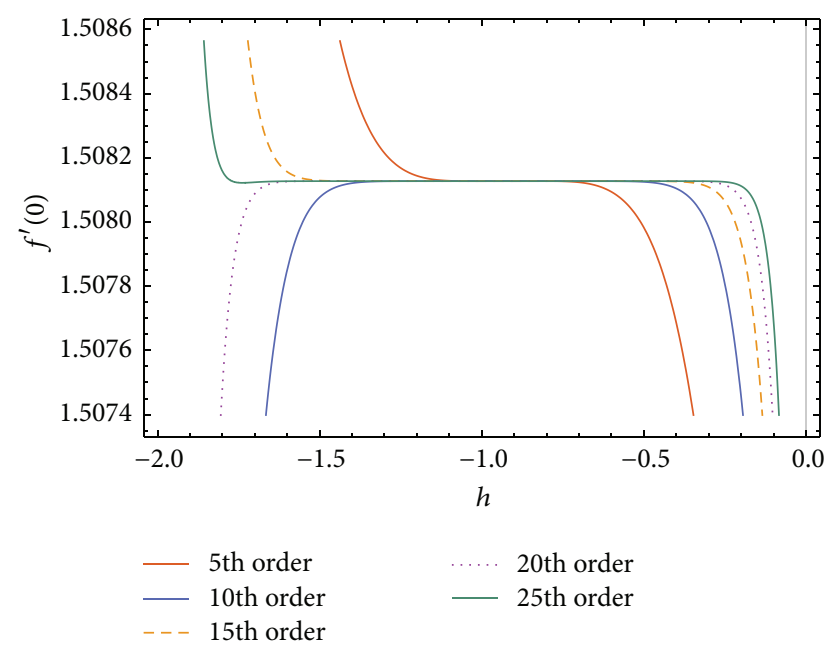

FIGURE 1: $h$-curve of different order solutions for $m_{h}=0.3, m_{p}=0.7$, and $R_{m p}=1$.

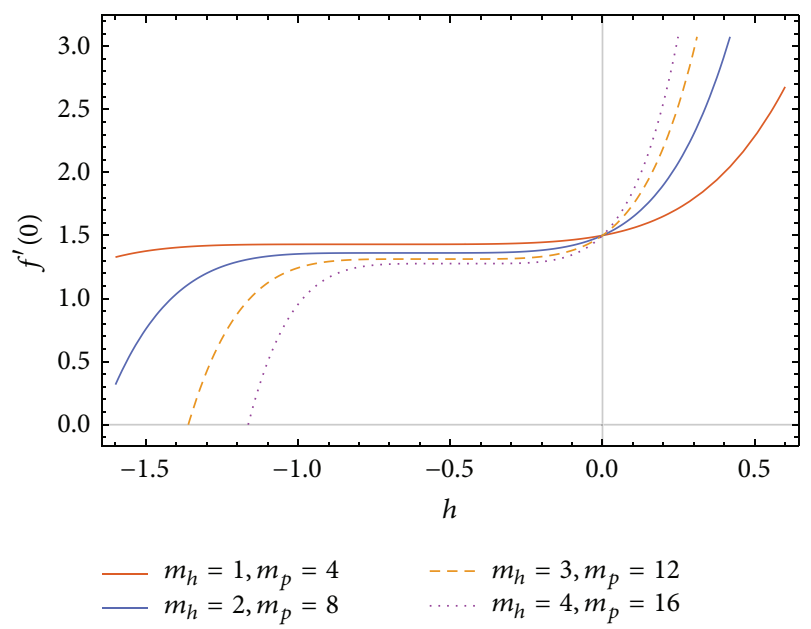

FIGURE 2: $h$-curve of fifth-order solution for various $m_{h}$ and $m_{p}$ and $R_{m p}=1$.

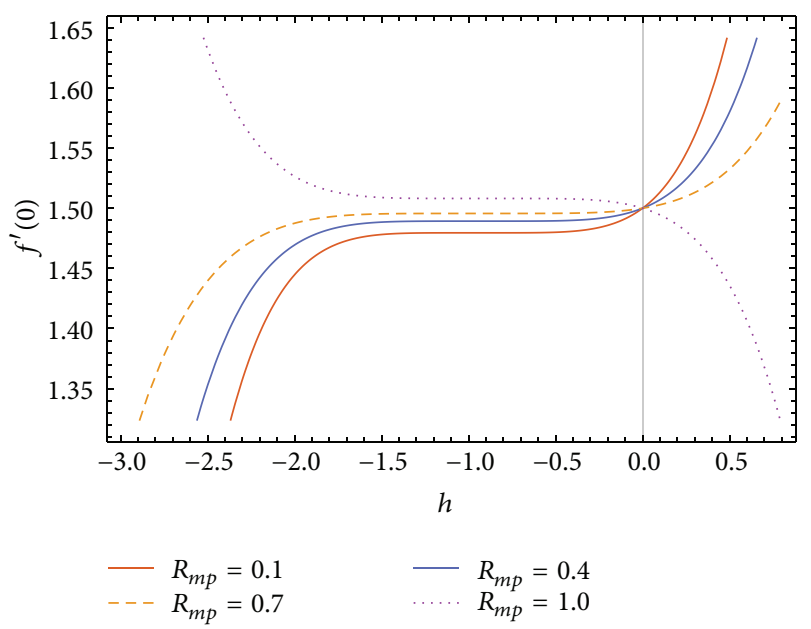

FIGURE 3: $h$-curve of fifth-order solution for various $R_{m p}$ and $m_{h}=$ 0.6 and $m_{p}=0.4$.

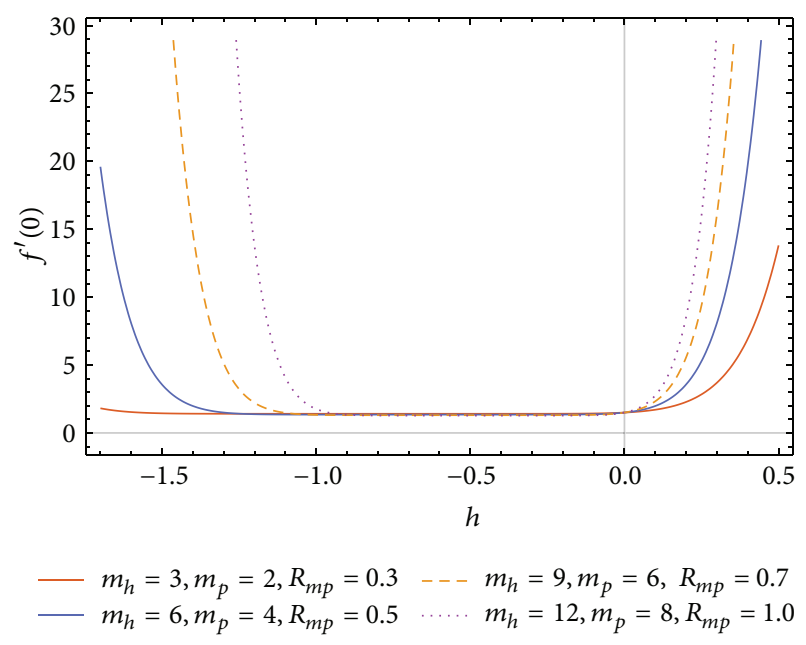

FIGURE 4: $h$-curve of tenth-order solution for various $m_{h}, m_{p}$, and $R_{m p}$.

values of $R_{m p}$ and for fixed values of $m_{h}, m_{p}$ are displayed in Table 2. Table 3 is constructed to display tenth-order absolute residuals for different values of $m_{h}, m_{p}$, and $h$ while keeping Reynold number fixed. Exact solution is obtained in case of zero Reynold number when the differential equation in (9) becomes linear. Fifth- and tenth-order HAM solutions are compared with this exact solution in Table 4 using the concept of absolute error. Keeping $h$ and Reynold and Hartmann numbers fixed, Table 5 displays different order HAM solutions. Table 6 shows important information about the consistency and efficiency of HAM by means of average absolute residuals for fifth-order approximation. Convergence of the present technique is given in Table 7 for different order approximations by means of $f^{\prime}(0),\left|f^{\prime \prime \prime}(0)\right|, f^{v}(0)$, and $\left|f^{v i i}(0)\right|$. As $f^{n}(0)$ becomes zero for even $n$, odd order derivatives are taken to study the convergence of the technique used. Comparison of different analytical techniques and one numerical Mathematica command NDSolve with the present work is displayed in Table 8 which shows that results obtained by HAM are in high agreement. One can refine these results by selecting suitable $h$ in the valid region $R_{h}$. The rapid convergence of HAM can also be seen in Figure 5 which shows the residuals of various analytical schemes.

\section{Conclusion}

All tables (Tables 1-8) show the efficiency and consistency of the mentioned scheme. The valid region $R_{h}$ can be more refined to choose such value of $h$ for which the obtained solution converges rapidly which is the beauty of the present analytic technique. It is also observed that while increasing the value of Hartmann numbers (Figures 2 and 4), the valid region moves towards the right. Table 3 also tells the same story. The influences of different parameters on the velocity profile are displayed in Figures 6-9 and the following observations are made:

(1) Velocity of fluid in porous medium and the Reynold number $R_{m p}$ are inversely proportional to each other; 
TABLE 2: Absolute residuals for different $R_{m p}$ keeping $m_{h}$ and $m_{p}$ fixed.

\begin{tabular}{llcccc}
\hline & & \multicolumn{2}{c}{ Fifth-order absolute residuals } & $R_{m p}=0.7$ & $R_{m p}=0.9$ \\
$z$ & $R_{m p}=0.1$ & $R_{m p}=0.3$ & $1.3902 \times 10^{-9}$ & $2.2210 \times 10^{-9}$ & $9.1597 \times 10^{-11}$ \\
0.1 & $1.2301 \times 10^{-10}$ & $2.2645 \times 10^{-9}$ & $2.2210 \times 10^{-9}$ & $5.9589 \times 10^{-10}$ & $8.9372 \times 10^{-9}$ \\
0.3 & $9.2250 \times 10^{-10}$ & $2.1661 \times 10^{-9}$ & $4.4410 \times 10^{-10}$ & $4.7810 \times 10^{-10}$ & $3.5040 \times 10^{-9}$ \\
0.4 & $2.8005 \times 10^{-9}$ & $6.2185 \times 10^{-10}$ & $2.2210 \times 10^{-9}$ & $1.2061 \times 10^{-9}$ & $9.3540 \times 10^{-8}$ \\
0.5 & $5.5909 \times 10^{-9}$ & $2.9842 \times 10^{-9}$ & $2.2210 \times 10^{-8}$ & $2.0999 \times 10^{-9}$ & $3.0340 \times 10^{-7}$ \\
0.6 & $8.2828 \times 10^{-9}$ & $8.9656 \times 10^{-9}$ & $4.4410 \times 10^{-8}$ & $3.2913 \times 10^{-9}$ & $6.0391 \times 10^{-7}$ \\
0.7 & $8.8277 \times 10^{-9}$ & $1.6855 \times 10^{-8}$ & $4.4410 \times 10^{-8}$ & $1.4133 \times 10^{-8}$ & $4.0297 \times 10^{-7}$ \\
0.8 & $3.9394 \times 10^{-9}$ & $2.4190 \times 10^{-8}$ & $2.2210 \times 10^{-10}$ & $3.2937 \times 10^{-8}$ & $1.1930 \times 10^{-7}$ \\
0.9 & $1.1760 \times 10^{-8}$ & $2.7364 \times 10^{-8}$ & $4.4410 \times 10^{-9}$ & $1.0928 \times 10^{-8}$ & $4.0659 \times 10^{-7}$ \\
1.0 & $4.9318 \times 10^{-8}$ & $2.5064 \times 10^{-8}$ & $2.2205 \times 10^{-8}$ & $1.3853 \times 10^{-7}$ & $5.5660 \times 10^{-6}$ \\
\hline
\end{tabular}

TABLE 3: Absolute residuals for different values of $m_{h}, m_{p}$ and $h$ keeping $R_{m p}=1$.

\begin{tabular}{|c|c|c|c|c|c|}
\hline \multicolumn{6}{|c|}{ Tenth-order absolute residuals } \\
\hline \multirow{3}{*}{$z$} & $m_{h}=0.5$ & $m_{h}=2.5$ & $m_{h}=3.5$ & $m_{h}=4.5$ & $m_{h}=5.5$ \\
\hline & $m_{p}=2.5$ & $m_{p}=3.5$ & $m_{p}=5.5$ & $m_{p}=7.5$ & $m_{p}=9.5$ \\
\hline & $h=-0.905$ & $h=-0.81$ & $h=-0.76$ & $h=-0.702$ & $h=-0.652$ \\
\hline 0.1 & $1.7770 \times 10^{-12}$ & $5.2593 \times 10^{-11}$ & $2.0127 \times 10^{-8}$ & $3.2010 \times 10^{-7}$ & $1.7200 \times 10^{-6}$ \\
\hline 0.2 & $2.2357 \times 10^{-12}$ & $2.7173 \times 10^{-10}$ & $3.7920 \times 10^{-8}$ & $6.4820 \times 10^{-7}$ & $3.9832 \times 10^{-6}$ \\
\hline 0.3 & $6.6970 \times 10^{-12}$ & $6.7927 \times 10^{-10}$ & $5.0219 \times 10^{-8}$ & $8.4460 \times 10^{-7}$ & $5.8732 \times 10^{-6}$ \\
\hline 0.4 & $1.2603 \times 10^{-11}$ & $1.0666 \times 10^{-9}$ & $5.3527 \times 10^{-8}$ & $5.7542 \times 10^{-7}$ & $4.2189 \times 10^{-6}$ \\
\hline 0.5 & $2.1863 \times 10^{-11}$ & $1.0404 \times 10^{-9}$ & $4.8000 \times 10^{-8}$ & $4.9288 \times 10^{-7}$ & $5.2428 \times 10^{-6}$ \\
\hline 0.6 & $3.3222 \times 10^{-11}$ & $2.0734 \times 10^{-10}$ & $4.8612 \times 10^{-8}$ & $2.0817 \times 10^{-6}$ & $2.2440 \times 10^{-5}$ \\
\hline 0.7 & $3.7364 \times 10^{-11}$ & $1.7351 \times 10^{-9}$ & $1.1400 \times 10^{-7}$ & $2.0580 \times 10^{-6}$ & $2.9649 \times 10^{-5}$ \\
\hline 0.8 & $2.6140 \times 10^{-11}$ & $5.6690 \times 10^{-9}$ & $4.1453 \times 10^{-7}$ & $6.0343 \times 10^{-6}$ & $3.7432 \times 10^{-5}$ \\
\hline 0.9 & $3.5145 \times 10^{-12}$ & $1.6370 \times 10^{-8}$ & $1.4760 \times 10^{-6}$ & $3.9871 \times 10^{-5}$ & $3.6410 \times 10^{-4}$ \\
\hline 1.0 & $2.8584 \times 10^{-11}$ & $5.7388 \times 10^{-8}$ & $5.2801 \times 10^{-6}$ & $1.5630 \times 10^{-4}$ & $1.5182 \times 10^{-3}$ \\
\hline
\end{tabular}

TABLE 4: Absolute errors of fifth- and tenth-order HAM solutions when $R_{m p}=0$.

\begin{tabular}{lccc}
\hline & & Absolute errors when $R_{m p}=0$ \\
$z$ & Exact solution & Fifth-order AE & Tenth-order AE \\
\hline 0.0 & 0 & 0 & 0 \\
0.1 & 0.147171 & $7.58785506649317 \times 10^{-10}$ & 0 \\
0.2 & 0.291618 & $1.39478356642186 \times 10^{-9}$ & $3.33066907387546 \times 10^{-16}$ \\
0.3 & 0.430586 & $1.80948145356296 \times 10^{-9}$ & $8.32667268468867 \times 10^{-16}$ \\
0.4 & 0.561271 & $1.94815263920844 \times 10^{-9}$ & $7.77156117237609 \times 10^{-16}$ \\
0.5 & 0.680780 & $1.81075676675135 \times 10^{-9}$ & $4.44089209850062 \times 10^{-16}$ \\
0.6 & 0.786113 & $1.45204859247627 \times 10^{-9}$ & $2.22044604925031 \times 10^{-16}$ \\
0.7 & 0.874125 & $9.70818092582703 \times 10^{-10}$ & $5.55111512312578 \times 10^{-16}$ \\
0.8 & 0.941500 & $4.90335771985428 \times 10^{-10}$ & $7.77156117237609 \times 10^{-16}$ \\
0.9 & 0.984713 & $1.33847599670389 \times 10^{-10}$ & $1.11022302462515 \times 10^{-16}$ \\
1.0 & 1.000000 & $2.22044604925031 \times 10^{-16}$ & $2.22044604925030 \times 10^{-16}$ \\
\hline
\end{tabular}

$\mathrm{AE}=\mid$ exact solution $-\mathrm{HAM}$ solution $\mid$.

that is, increasing Reynold number (when density of fluid increases) results in the decrease in velocity of fluid while keeping Hartmann number fixed as shown in Figure 6.

(2) Figure 7 shows that while increasing $m_{h}$ (i.e., increasing the imposed magnetic effect) and keeping $m_{p}$, $R_{m p}$ fixed, the velocity of fluid increases.
(3) The same effect is studied in Figure 8 by increasing $m_{p}$ (i.e., when the permeability decreases) and keeping $m_{h}$ and $R_{m p}$ fixed.

(4) Increasing Hartmann and Reynold numbers together, it is observed from Figure 9 that the velocity of fluid increases. It is concluded that Hartmann number is more influential as compared to Reynold number. 
TABLE 5: Different order solutions keeping $m_{h}=m_{p}=0.5, R_{m p}=1$, and $h=-0.97$.

\begin{tabular}{|c|c|c|c|c|c|}
\hline \multicolumn{6}{|c|}{ Different order HAM solutions } \\
\hline$z$ & 1st order & 3rd order & 5th order & 15 th order & 25th order \\
\hline 0.0 & 0 & 0 & 0 & 0 & 0 \\
\hline 0.1 & 0.15034713893 & 0.15029407026 & 0.15029422756 & 0.15029422727 & 0.15029422694 \\
\hline 0.2 & 0.29758357211 & 0.29748028081 & 0.29748058054 & 0.29748058002 & 0.29748057936 \\
\hline 0.3 & 0.43861285278 & 0.43846608333 & 0.43846649504 & 0.43846649440 & 0.43846649341 \\
\hline 0.4 & 0.57036617920 & 0.57018808886 & 0.57018856454 & 0.57018856394 & 0.57018856267 \\
\hline 0.5 & 0.68981403459 & 0.68962312433 & 0.68962359743 & 0.68962359707 & 0.68962359568 \\
\hline 0.6 & 0.79397520822 & 0.79379541675 & 0.79379580965 & 0.79379580971 & 0.79379580853 \\
\hline 0.7 & 0.87992232433 & 0.87977913949 & 0.87977938815 & 0.87977938864 & 0.87977938832 \\
\hline 0.8 & 0.94478300617 & 0.94469588175 & 0.94469597496 & 0.94469597563 & 0.94469597737 \\
\hline 0.9 & 0.98573580197 & 0.98570687775 & 0.98570688141 & 0.98570688178 & 0.98570688792 \\
\hline 1.0 & 0.99999999999 & 1.00000000000 & 1.00000000000 & 1.00000000000 & 1.00000001530 \\
\hline
\end{tabular}

TABLE 6: Average absolute residuals for various values of $m_{h}, m_{p}, R_{m p}$, and $h$.

\begin{tabular}{lccccc}
\hline & & \multicolumn{2}{c}{ Fifth-order average absolute residuals } \\
$m_{h}$ & $m_{p}$ & $R_{m p}$ & $h$ & Solution & Residual \\
\hline 0.3 & 0.7 & 1.0 & -0.972 & 0.6896235970708491 & $3.385181024 \times 10^{-12}$ \\
1.2 & 0.8 & 0.7 & -0.685 & 0.5614010061254293 & $5.195453594 \times 10^{-7}$ \\
3.0 & 2.0 & 0.5 & -0.773 & 0.8625404743084730 & $1.845019426 \times 10^{-8}$ \\
3.5 & 4.5 & 0.9 & -0.851 & 0.6537531470383126 & $9.278417177 \times 10^{-8}$ \\
6.0 & 4.0 & 1.0 & -0.597 & 0.7562438682896610 & $5.552400535 \times 10^{-5}$ \\
10.0 & 5.0 & 1.5 & -0.656 & 0.7456786847598219 & $1.257868035 \times 10^{-5}$ \\
12.0 & 8.0 & 2.0 & -0.590 & 0.6266880364547981 & $2.172601809 \times 10^{-5}$ \\
15.0 & 10.0 & 2.5 & -0.520 & 0.7309895729856828 & $4.155602273 \times 10^{-4}$ \\
\hline
\end{tabular}

TABLE 7: Convergence of HAM for different order approximations.

\begin{tabular}{lcccc}
\hline Approximation order & $f^{\prime}(0)$ & $\left|f^{\prime \prime \prime}(0)\right|$ & $f^{v}(0)$ & $\left|f^{(v i i)}(0)\right|$ \\
\hline 1 & 0.00866 & 3.11432 & 1.45499 & 8.729999 \\
3 & 0.00812 & 3.11177 & 1.58119 & 1.58118 \\
5 & 0.00812 & 3.11178 & 1.58118 & 15.22983 \\
7 & 0.00812 & 3.11178 & 1.58118 & 15.255853 \\
10 & 0.00812 & 3.11178 & 1.58118 & 15.25593 \\
15 & 0.00812 & 3.11178 & 1.58118 & 15.25593 \\
20 & 0.00812 & 3.11178 & 1.58118 & 15.25593 \\
25 & 0.00812 & 3.11178 & & 15.25595 \\
\hline
\end{tabular}

TABLE 8: Comparison of HAM with numerical and other analytical techniques.

\begin{tabular}{|c|c|c|c|c|c|c|}
\hline \multicolumn{7}{|c|}{ Absolute residuals for numerical and different analytical schemes } \\
\hline$z$ & NDSolve & DTM & DJM & $\mathrm{ADM}$ & OHAM & HAM \\
\hline 0 & $3.47 \times 10^{-3}$ & 0 & 0 & 0 & 0 & 0 \\
\hline 0.1 & $3.20 \times 10^{-4}$ & $2.36 \times 10^{-13}$ & $2.86 \times 10^{-12}$ & $3.20 \times 10^{-5}$ & $1.51 \times 10^{-7}$ & 0 \\
\hline 0.2 & $2.02 \times 10^{-4}$ & $1.21 \times 10^{-10}$ & $3.56 \times 10^{-10}$ & $2.51 \times 10^{-4}$ & $2.72 \times 10^{-7}$ & $2.22 \times 10^{-16}$ \\
\hline 0.3 & $4.57 \times 10^{-5}$ & $4.62 \times 10^{-9}$ & $5.81 \times 10^{-9}$ & $8.30 \times 10^{-4}$ & $3.31 \times 10^{-7}$ & $4.44 \times 10^{-16}$ \\
\hline 0.4 & $5.67 \times 10^{-5}$ & $6.11 \times 10^{-8}$ & $4.09 \times 10^{-8}$ & $2.00 \times 10^{-3}$ & $3.05 \times 10^{-7}$ & 0 \\
\hline 0.5 & $1.51 \times 10^{-5}$ & $4.51 \times 10^{-7}$ & $1.79 \times 10^{-7}$ & $3.51 \times 10^{-3}$ & $1.75 \times 10^{-7}$ & 0 \\
\hline 0.6 & $3.45 \times 10^{-5}$ & $2.31 \times 10^{-6}$ & $5.78 \times 10^{-7}$ & $5.70 \times 10^{-3}$ & $6.62 \times 10^{-8}$ & $4.44 \times 10^{-16}$ \\
\hline 0.7 & $5.07 \times 10^{-7}$ & $9.12 \times 10^{-6}$ & $1.50 \times 10^{-6}$ & $8.33 \times 10^{-3}$ & $4.05 \times 10^{-7}$ & $4.44 \times 10^{-16}$ \\
\hline 0.8 & $4.56 \times 10^{-5}$ & $3.00 \times 10^{-5}$ & $3.25 \times 10^{-6}$ & $1.12 \times 10^{-2}$ & $8.08 \times 10^{-7}$ & $4.44 \times 10^{-16}$ \\
\hline 0.9 & $1.20 \times 10^{-4}$ & $8.50 \times 10^{-5}$ & $6.17 \times 10^{-6}$ & $1.41 \times 10^{-2}$ & $1.23 \times 10^{-6}$ & 0 \\
\hline 1.0 & $6.99 \times 10^{-4}$ & $2.20 \times 10^{-4}$ & $1.03 \times 10^{-5}$ & $1.64 \times 10^{-2}$ & $1.61 \times 10^{-6}$ & $2.22 \times 10^{-15}$ \\
\hline
\end{tabular}




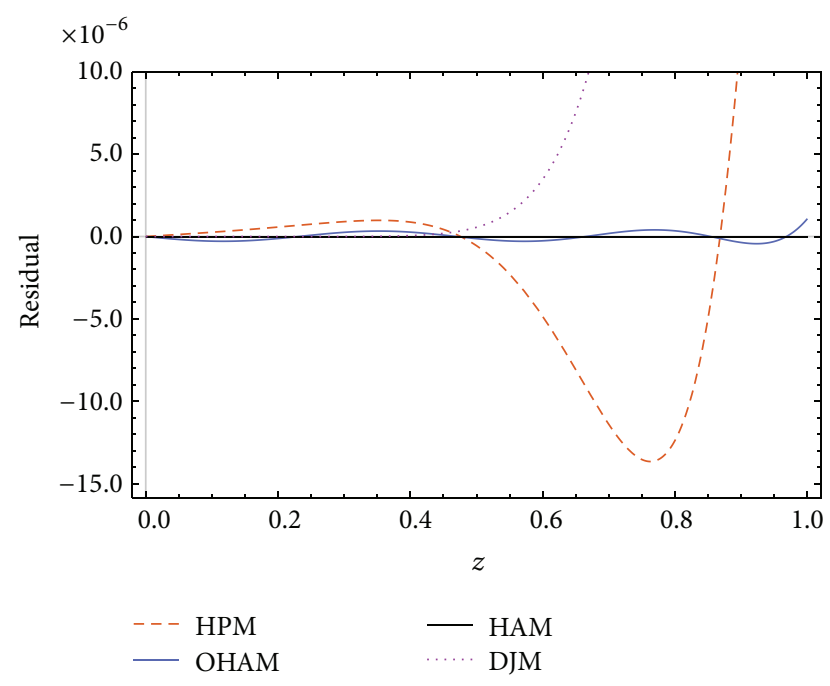

Figure 5: Comparison of HAM with other analytical schemes through residual.

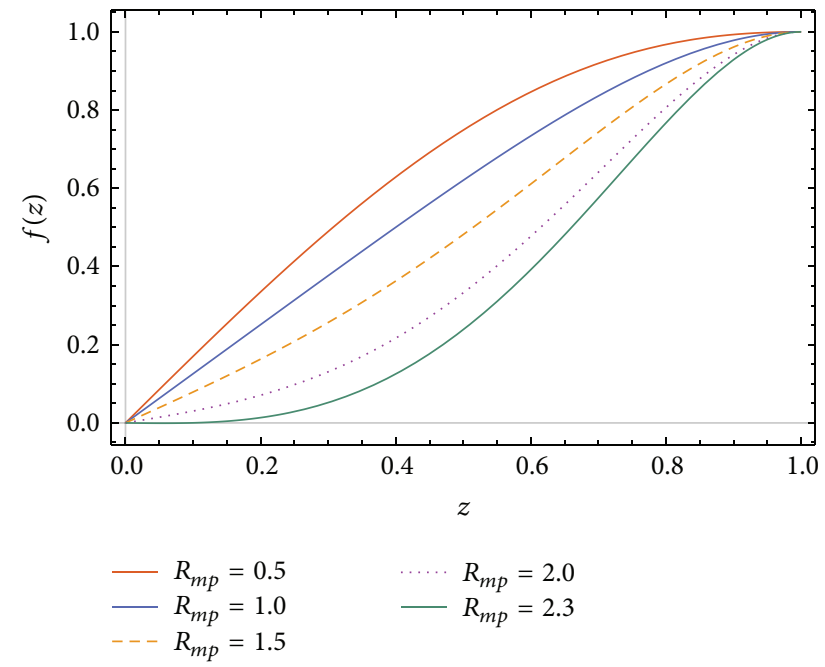

FIGURE 6: Velocity profile for different values of $R_{m p}$ with $m_{h}=m_{p}=$ 0.5 .

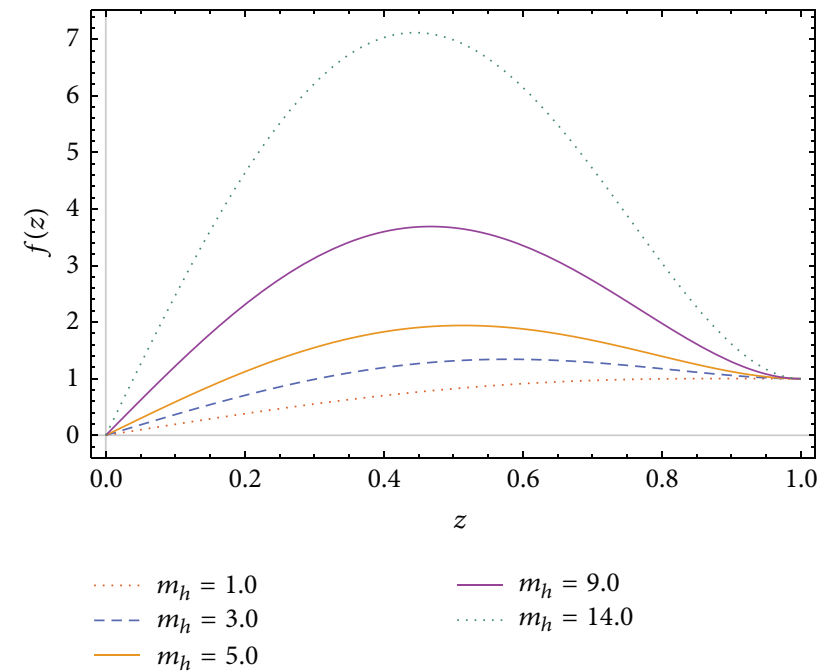

FIGURE 7: Velocity profile for different values of $m_{h}$ with $m_{p}=R_{m p}=$ 1.

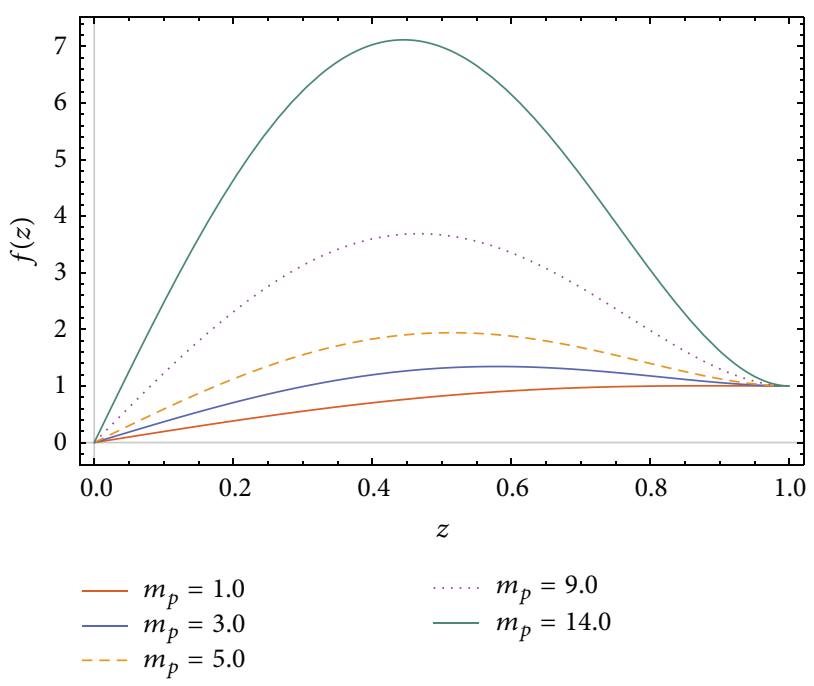

FIGURE 8: Velocity profile for different values of $m_{p}$ with $m_{h}=R_{m h}=$ 1.

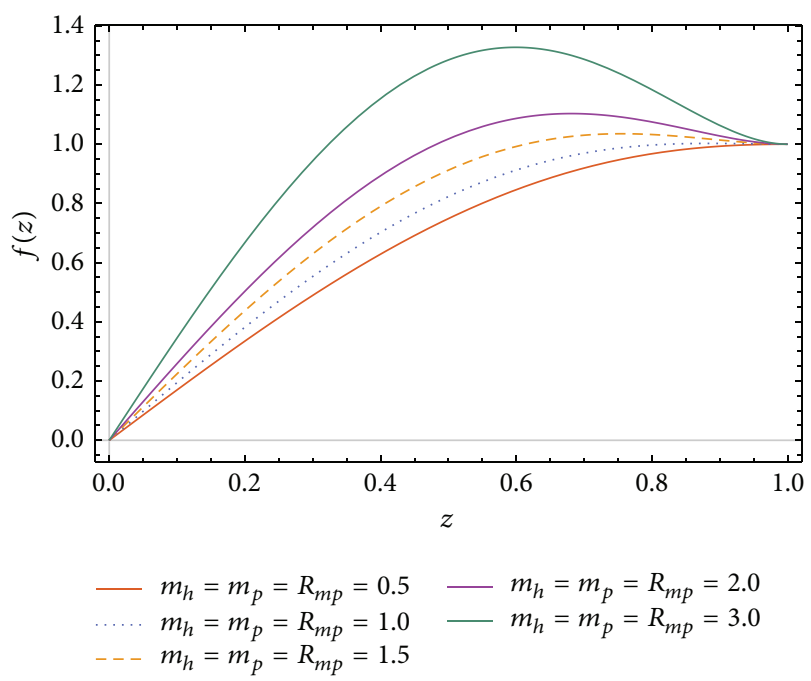

FIGURE 9: Velocity profile for equal values of $m_{p}, m_{h}$, and $R_{m p}$.

\section{Competing Interests}

The authors declare that they have no competing interests.

\section{References}

[1] W. Tan and T. Masuoka, "Stokes' first problem for a second grade fluid in a porous half-space with heated boundary," International Journal of Non-Linear Mechanics, vol. 40, no. 4, pp. 515$522,2005$.

[2] B. Y. Jamaloei, "A review of recent advances in the study of drainage in porous media," Special Topics and Reviews in Porous Media, vol. 4, no. 3, pp. 253-272, 2013.

[3] K. Vafai, Handbook of Porous Media, CRC Press, New York, NY, USA, 2015.

[4] N. B. Naduvinamani, P. S. Hiremath, and G. Gurubasavaraj, "Squeeze film lubrication of a short porous journal bearing with 
couple stress fluids," Tribology International, vol. 34, no. 11, pp. 739-747, 2001.

[5] M. Qayyum, H. Khan, M. T. Rahim, I. Ullah, and M. Dao, "Modeling and analysis of unsteady axisymmetric squeezing fluid ow through porous medium channel with slip boundary," PLoS ONE, vol. 10, no. 3, Article ID e0117368, 2015.

[6] Samiulhaq, S. Ahmad, D. Vieru, I. Khan, and S. Shafie, "Unsteady magnetohydrodynamic free convection flow of a second grade fluid in a porous medium with ramped wall temperature," PLoS ONE, vol. 9, no. 5, Article ID e88766, 2014.

[7] A. Sohail, Samiulhaq, and D. Vieru, "Unsteady free convection flow near an infinite vertical plate with prescribed surface heat flux and skin friction," The European Physical Journal Plus, vol. 129, no. 2, article 28, 7 pages, 2014.

[8] S. J. Liao, The proposed homotopy analysis technique for the solution of nonlinear problems [Ph.D. thesis], Shanghai Jiao Tong University, 1992.

[9] S. J. Liao, "On the relationship between the homotopy analysis method and Euler transform," Communications in Nonlinear Science and Numerical Simulation, vol. 15, no. 6, pp. 1421-1431, 2010.

[10] S. Abbasbandy, E. Shivanian, and K. Vajravelu, "Mathematical properties of $\hbar$-curve in the frame work of the homotopy analysis method," Communications in Nonlinear Science and Numerical Simulation, vol. 16, no. 11, pp. 4268-4275, 2011.

[11] S. Abbasbandy, "Homotopy analysis method for the Kawahara equation," Nonlinear Analysis: Real World Applications, vol. 11, no. 1, pp. 307-312, 2010.

[12] T. Hayat, M. Mustafa, and S. Asghar, "Unsteady flow with heat and mass transfer of a third grade fluid over a stretching surface in the presence of chemical reaction," Nonlinear Analysis. Real World Applications, vol. 11, no. 4, pp. 3186-3197, 2010.

[13] S. J. Liao, Beyond Perturbation: Introduction to Homotopy Analysis Method, Chapman and Hall/CRC, 2003.

[14] F. Mabood and W. A. Khan, "Approximate analytic solutions for influence of heat transfer on MHD stagnation point flow in porous medium," Computers \& Fluids, vol. 100, pp. 72-78, 2014.

[15] F. Mabood and W. A. Khan, "Homotopy analysis method for boundary layer flow and heat transfer over a permeable flat plate in a Darcian porous medium with radiation effects," Journal of the Taiwan Institute of Chemical Engineers, vol. 45, no. 4, pp. 1217-1224, 2014.

[16] M. M. Rashidi, H. Shahmohamadi, and S. Dinarvand, "Analytic approximate solutions for unsteady two-dimensional and axisymmetric squeezing flows between parallel plates," Mathematical Problems in Engineering, vol. 2008, Article ID 935095, 12 pages, 2008.

[17] M. Mustafa, T. Hayat, and S. Obaidat, "On heat and mass transfer in the unsteady squeezing flow between parallel plates," Meccanica, vol. 47, no. 7, pp. 1581-1589, 2012.

[18] C. K. Kirubhashankar and S. Ganesh, "Unsteady MHD flow of a casson fluid in a parallel plate channel with heat and mass transfer of chemical reaction," Paripex-Indian Journal of Research, vol. 3, no. 2, 2014.

[19] A. M. Siddiqui, S. Irum, and A. R. Ansari, "Unsteady squeezing flow of a viscous MHD fluid between parallel plates, a solution using the homotopy perturbation method," Mathematical Modelling and Analysis, vol. 13, no. 4, pp. 565-576, 2008.

[20] A. M. Siddiqui, S. Irum, and A. R. Ansari, "Unsteady squeezing flow of a viscous MHD fluid between parallel plates, a solution using the homotopy perturbation method," Mathematical Modelling and Analysis, vol. 13, no. 4, pp. 565-576, 2008.
[21] S. Ganesh and S. Krishnambal, "Unsteady stokes flow of viscous fluid between two parallel porous plates," Journal on Information Sciences and Computing, vol. 1, no. 1, pp. 63-66, 2007.

[22] A. Mohamed Ismail, S. Ganesh, and C. K. Kirubhashankar, "Unsteady Magnetohydrodynamic flow between two Parallel Plates through a porous medium," International Journal on Design and Manufacturing Technologies, vol. 7, no. 2, pp. 1-6, 2013.

[23] S. Abbasbandy and E. Shevanian, "Solution of singular linear vibrational BVPs by the homotopy analysis method," Journal of Numerical Mathematics and Stochastics, vol. 1, no. 1, pp. 77-84, 2009.

[24] M. Shaban, E. Shivanian, and S. Abbasbandy, "Analyzing magneto-hydrodynamic squeezing flow between two parallel disks with suction or injection by a new hybrid method based on the Tau method and the homotopy analysis method," The European Physical Journal Plus, vol. 128, article 133, 2013.

[25] H. Vosoughi, E. Shivanian, and S. Abbasbandy, "Unique and multiple PHAM series solutions of a class of nonlinear reactive transport model," Numerical Algorithms, vol. 61, no. 3, pp. 515524, 2012.

[26] E. Shivanian, H. H. Alsulami, M. S. Alhuthali, and S. Abbasbandy, "Predictor homotopy analysis method (Pham) for nano boundary layer flows with nonlinear Navier boundary condition: existence of four solutions," Filomat, vol. 28, no. 8, pp. 1687-1697, 2014.

[27] I. Ullah, M. T. Rahim, and H. Khan, "Application of Daftardar Jafari method to first grade MHD squeezing fluid flow in a porous medium with slip boundary condition," Abstract and Applied Analysis, vol. 2014, Article ID 479136, 8 pages, 2014. 


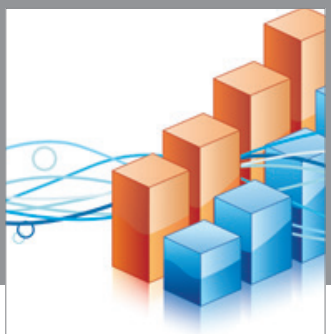

Advances in

Operations Research

vatem alat4

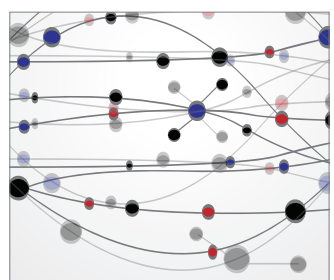

\section{The Scientific} World Journal
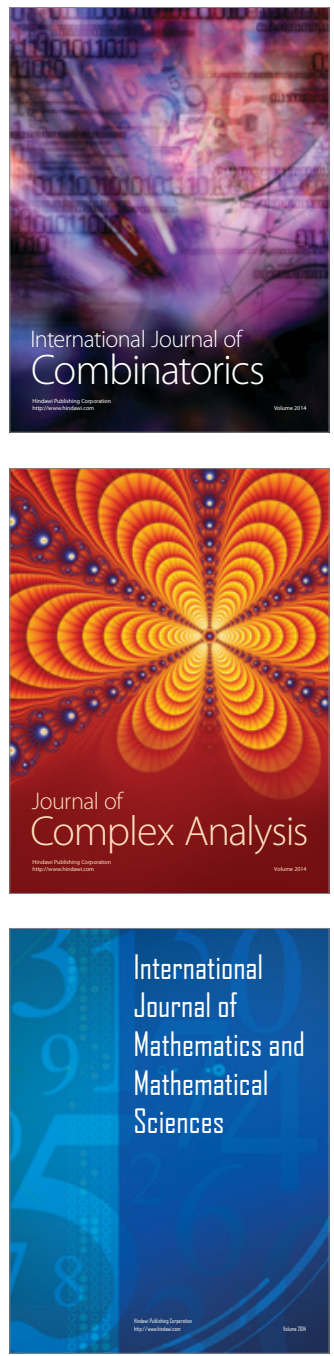
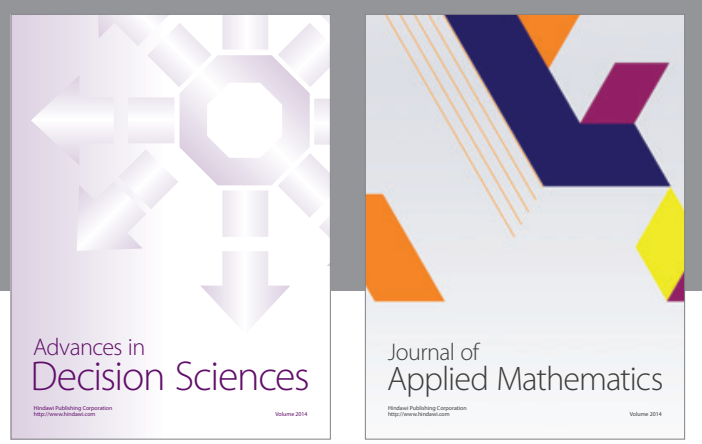

Algebra

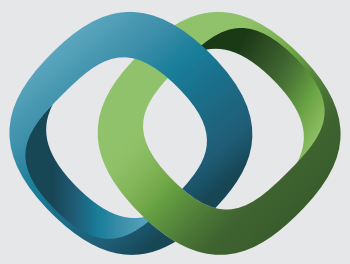

\section{Hindawi}

Submit your manuscripts at

http://www.hindawi.com
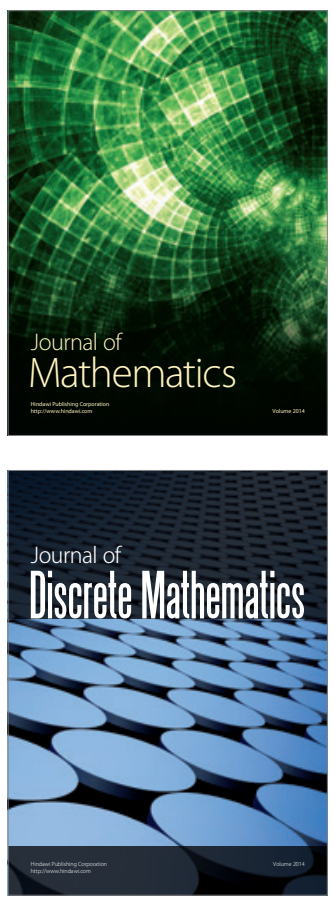

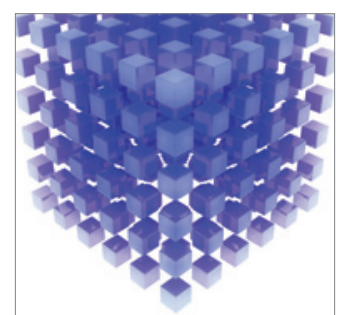

Mathematical Problems in Engineering
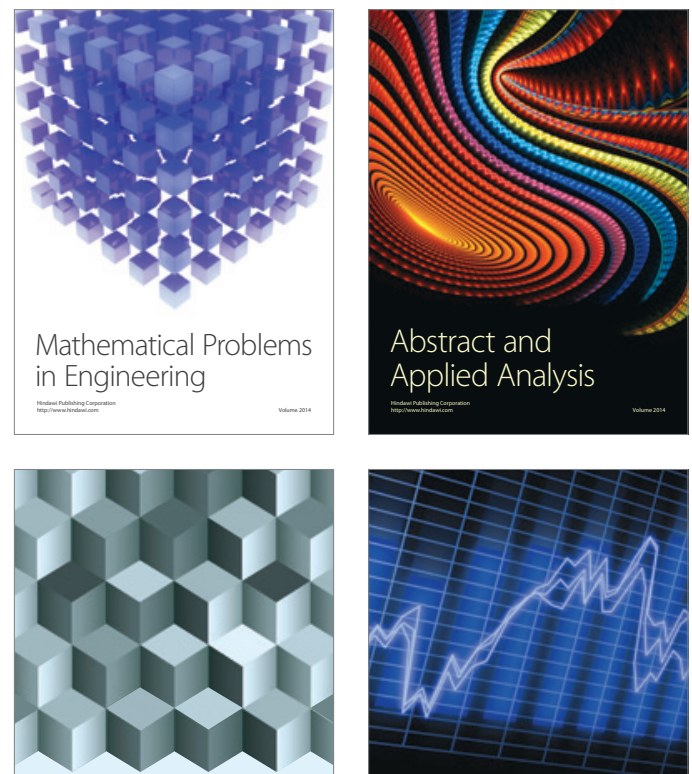

Journal of

Function Spaces

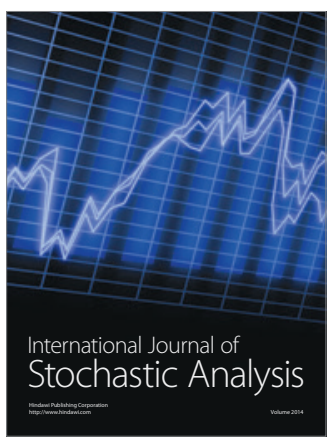

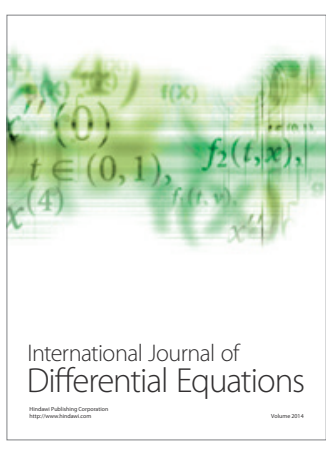
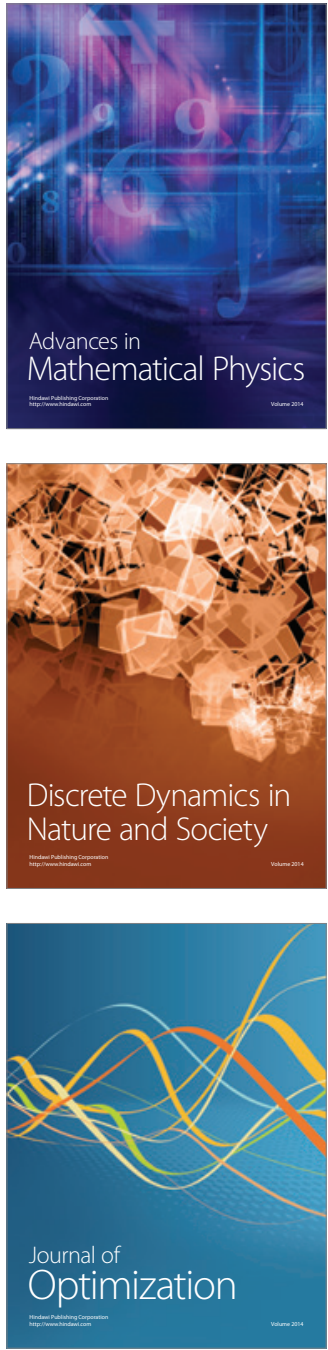